\title{
Quantitative Analysis of Viral Nanomachines in Liquid
}

\author{
A. Cameron Varano ${ }^{1,2}$, Madeline J. Dukes ${ }^{3}$, Sarah M. McDonald ${ }^{1}$, Steven Poelzing ${ }^{1}$, and \\ Deborah F. Kelly ${ }^{1}$ \\ ${ }^{1}$ Virginia Tech Carilion Research Institute, Virginia Tech, Roanoke, VA, 24016, USA. \\ ${ }^{2}$ Translation Biology, Medicine and Health, Virginia Tech, Blacksburg, VA, 24061, USA. \\ ${ }^{3}$ Protochips, Inc., Application Science, Raleigh, NC, 27560, USA.
}

Currently, there remains a critical need to develop innovative techniques for visualizing active biological processes at the nanoscale. In situ Transmission Electron Microscopy (TEM) provides the technical framework to address this critical need. Using a liquid cell platform, we developed a unique strategy to illuminate the dynamic movements of viral pathogens. We used rotavirus as a model system for these analyses due its established size, features, and chemically inducible enzymatic activity in vitro.

Employing the Poseidon 210 liquid specimen holder, we tethered enzymatically-activated rotavirus particles within the device [1] for TEM imaging and quantitative analysis (Figure 1a c). We collected image series of the dynamic virus particles at intervals of $\sim 10$ frames per seconds under low-dose conditions $\left(0.1\right.$ electrons $\left./ \AA^{2}\right)$ at $120 \mathrm{kV}$. At a magnification of $60,000 \times$, the final sampling in the output images was $5 \AA$ pixel at the specimen level. Individual virus particles were selected from the compiled image stacks and segmented accordingly as previously described [2]. The selected particles were compiled into separate movies using the iMovie software package with each movie representing a total time period of 10 seconds.

The resulting time-resolved movies revealed new dynamic information about the rotavirus particles contained in a liquid environment. We analyzed the mobility of the particles by applying a $3 \sigma$ density threshold to compute contour maps (Figure 1d). The contour images were also compiled to produce real-time movies. These movies showed the inherent mobility in the internal viral RNA genome and associated proteins. Additionally, we quantified the mobile units in relation to external mRNAs protruding from the viral capsid. The lengths of mRNA strands that surrounded the individual particles highly correlated with the inner mobility measurements. To computationally verify changes in particle mobility, 3D density maps were computed using standard routines [3]. We compared structures of virus samples prepared in solution to structures of frozen-hydrated samples. Structures of particles comprising the liquid specimens lacked the rigidity seen in the cryo-EM reconstructions (Figure 2). Collectively, our liquid cell imaging strategy, used in combination with cryo-EM, provides a more complete view of virus entities when assessing their active architectures [4]. The authors acknowledge the following funding sources that supported this work, 1R21AI113402-01 NIAID/NIH (D.F.K., S.M.M) and 1R01AI116815-01 NIAID/NIH (D.F.K., S.M.M).

\section{References:}

[1] Dukes M.J. et al., Microsc. Micoanal 20 (2014), pp. 338-345.

[2] Varano A.C., Rahimi A., Dukes M.J., Poelzing S., McDonald S.M., and Kelly D.F., ChemComm. 51 (2015), pp. 16176-16179.

[3] Frank J. et al., J. Struct Biol. 116 (1996), pp. 190-199.

[4] Rahimi A., Varano A.C., Demmert A.C., Melanson L.A., McDonald S.M., and Kelly D.F., J. Analyt. Molecul. Tech. 2 (2015), pp. 1-6. 

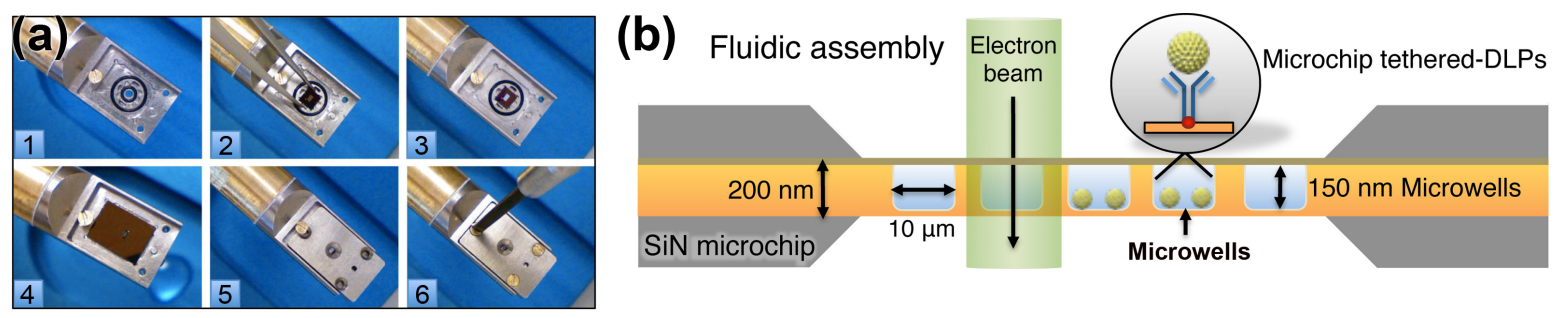

(c)
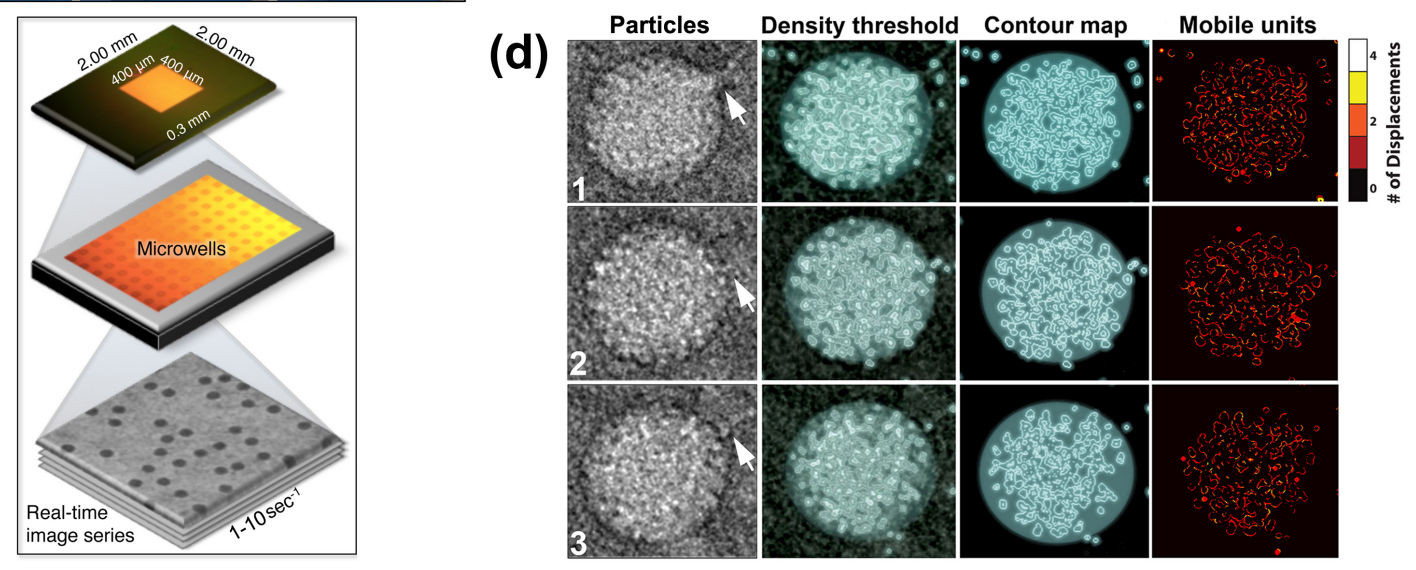

Figure 1. (a) The Poseidon 210 liquid specimen holder was assembled by placing O-ring fittings in the empty tip of the holder followed by the microchip assembly. A larger flat chip was placed over top to complete the assembly. A metal face-plate held in place by 3 brass screws hermetically sealed the assembly. (b) A cross-section illustration of the specimen holder positioned in the EM beam. The base chip of the liquid cell assembly includes a transparent imaging window consisting of integrated microwells $(10 \times 10$ microns $)$. (c) Activated rotavirus particles were tethered to the microwells and real-time image series were recorded in the TEM. (d) Virus particles were subjected to a density threshold filter. Contour maps were generated based on a $3 \sigma$-cutoff. The mobility of the virus particles is depicted in the final column. The heat map scales from 0 to 4 pixel displacements over a 10 second time frame. Panel width is 120 nm. Adapted from Varano et al., 2015 [2].

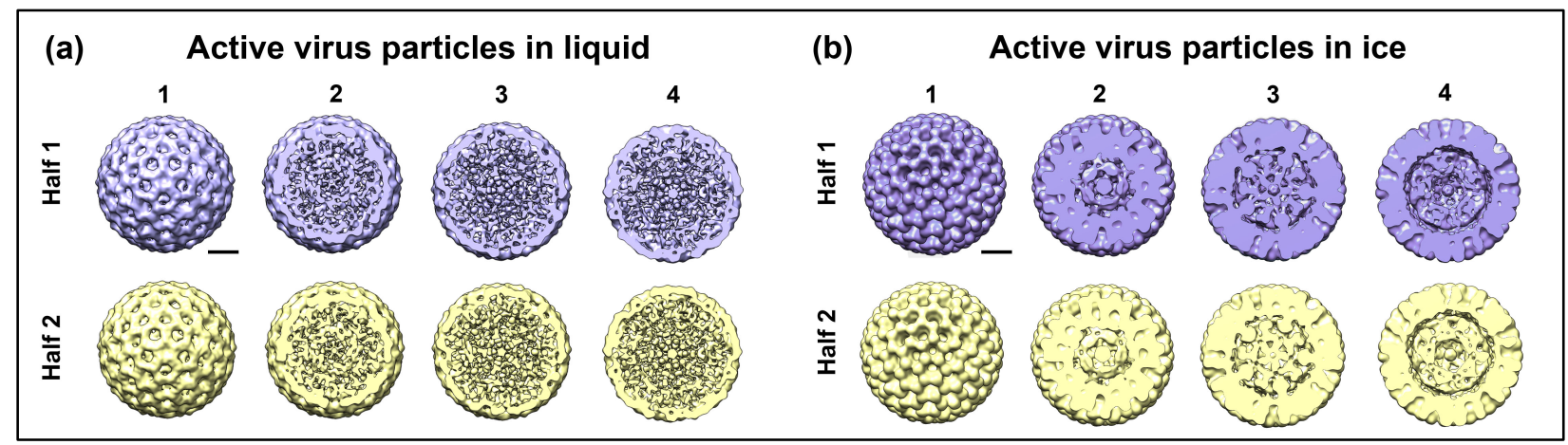

Figure 2. $3 \mathrm{D}$ reconstructions of active virus particles were calculated while enforcing icosahedral symmetry. Structures of virus particles in liquid (a) showed more dynamic internal features than structures of frozen-hydrated virus particles (b). Scale bar is $20 \mathrm{~nm}$. Adapted from Rahimi et al., 2015 [4]. 